\title{
Gas Leak
}

National Cancer Institute

\section{Source}

National Cancer Institute. Gas Leak. NCI Thesaurus. Code C63127.

Problem associated with the unintended escape of a gas from the container in which it is housed. 